\title{
Crystal fields and exchange in dilute alloys of Tb, Dy, and Er in Y studied for different concentrations
}

\section{Høg, J.; Touborg, P.}

\section{Published in:}

Physical Review B

Link to article, DOI:

10.1103/PhysRevB.11.520

Publication date:

1975

Document Version

Publisher's PDF, also known as Version of record

Link back to DTU Orbit

Citation $(A P A)$ :

Høg, J., \& Touborg, P. (1975). Crystal fields and exchange in dilute alloys of Tb, Dy, and Er in Y studied for different concentrations. Physical Review B, 11(1), 520-529. https://doi.org/10.1103/PhysRevB.11.520

\section{General rights}

Copyright and moral rights for the publications made accessible in the public portal are retained by the authors and/or other copyright owners and it is a condition of accessing publications that users recognise and abide by the legal requirements associated with these rights.

- Users may download and print one copy of any publication from the public portal for the purpose of private study or research.

- You may not further distribute the material or use it for any profit-making activity or commercial gain

- You may freely distribute the URL identifying the publication in the public portal 


\title{
Crystal fields and exchange in dilute alloys of $\mathrm{Tb}, \mathrm{Dy}$, and $\mathrm{Er}$ in $\mathrm{Y}$ studied for different concentrations
}

\author{
J. Høg and P. Touborg \\ Department of Electrophysics, Technical University, 2800 Lyngby, Denmark
}

(Received 22 May 1974)

\begin{abstract}
This is a sequel to an earlier paper reporting magnetization measurements on $\mathrm{Y}-0.14-$ at. \%-Dy and Y-0.14-at. \%-Er. In the present paper we have performed similar measurements on different concentrations of dilute Er, Dy, and $\mathrm{Tb}$ alloys and also included specific measurements of the basal-plane anisotropy. Crystal-field and exchange parameters were obtained by fits to the inverse initial susceptibility. The anisotropy measurements provide a confirmation of the crystal-field parameters in Dy and $\mathrm{Er}$ and are used to deduce $B_{66}$ in $\mathrm{Tb}$. The measurements on different concentrations confirm the adequacy of the theoretical models used. The crystal-field parameters vary substantially with the solute. The molecular-field tensors are highly anisotropic and are approximately independent of concentration at small concentrations.
\end{abstract}

\section{INTRODUCTION}

In a previous paper ${ }^{1}$ we have reported static magnetization measurements on $\mathrm{Y}-0.14 \%$-Er and $\mathrm{Y}-0.14 \%$-Dy (in the present paper \% means atomic $\%)$. The study of these dilute alloys was initiated to determine the crystalline electric fields, which are difficult to obtain from measurements on the pure rare-earth metals. As a continuation of this work-from which an unexpected behavior of the crystal-field parameters was deduced-we report in the present paper magnetization measurements on dilute $\mathrm{Y}-\mathrm{Tb}, \mathrm{Y}-\mathrm{Dy}$, and $\mathrm{Y}-\mathrm{Er}$ single crystals. These measurements were performed on crystals of different concentrations to check the model used, in which the crystal-field parameters do not depend on the concentration. The measurements also eliminate effects of spurious impurities in the host metal and elucidate the dependence of the exchange interactions on the concentration. The appropriate concentrations for such experiments may thereby be deduced. To check the parameters further we have performed separate measurements of the basal-plane anisotropy. In the case of $\mathrm{Tb}$ these measurements were necessary to obtain all the crystal-field parameters unambiguously.

In the following, we describe the experimental and theoretical procedures used in this investigation. The experimental results are then presented for each solute separately and finally the results are discussed.

\section{EXPERIMENTAL PROCEDURE}

The single-crystal samples, including a pure $\mathrm{Y}$ sample, were grown by the techniques of annealing arc-melted buttons. ${ }^{2}$ The materials used were $99.99 \%$ for the $\mathrm{Y}$ and $99.9 \%$ for the $\mathrm{Tb}, \mathrm{Dy}$, and Er. The measurements were performed using three different magnetometers, a Faraday magnetometer (FM), ${ }^{1}$ a $5-\mathrm{Hz}$ vibrating sample magnetometer (VSM), ${ }^{1}$ and a $169-\mathrm{Hz}$ vibrating sample anisotropy meter (VSA). ${ }^{3}$ All magnetometers were mounted in cryostats with superconducting magnets. The very sensitive Faraday magnetometer was used mainly for measuring initial susceptibilities, while the vibrating sample magnetometer was used for magnetization measurements at higher fields. The absolute accuracy of the magnetic moment measured in these two magnetometers was better than $1 \%$, and the fields were known accurately within $0.5 \%$. The Faraday and the vibrating sample magnetometers are described in further details in Ref. 1. The vibrating sample anisotropy meter was used for measuring the basal-plane anisotropy. In this apparatus the horizontal moment components parallel, $M_{11}$, and perpendicular, $M_{\perp}$, to the magnetic field were measured as a function of crystal rotation angle $\varphi$ about the vertical $c$ axis. $M_{\perp}$, which gives a sensitive measure of the anisotropy, was recorded as the crystal was rotated in steps of $2.5^{\circ}$. A typical output is shown in Fig. 1. $M_{\perp}$ in these experiments was always smaller than $1 \%$ of the magnitude of total moment. $M_{\perp}=M_{\perp}(\varphi)$ was Fourier analyzed giving the harmonic components of order $6 p, p=1,2, \ldots$, expected for a crystal with hexagonal symmetry. Other components of order 0 , 1 , 2, etc., are present. Some of these harmonics are error signals arising from geometrical effects like nonalignment, etc., of the magnetometer. The second-order component stems from the $c$ axis not being exactly vertical or the samples not being ideal spheres. The errors in the hexagonal harmonics are estimated from the size of the fourth, fifth, seventh, etc., order components.

The samples measured in the anisotropy meter had to be kept below $2.5 \mathrm{~mm}$ in diameter. These 


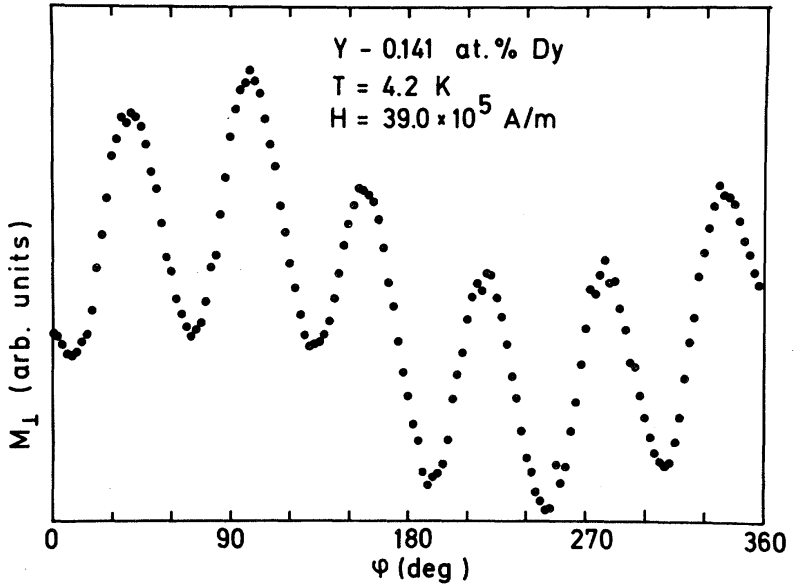

FIG. 1. Typical VSA recording of the perpendicular magnetization. The origin on the $\varphi$ axis is arbitrary.

small samples were cut from the same crystals as the approximately $3.5-\mathrm{mm}$ spheres used for the other magnetometers. To take account of inhomogeneities, the concentrations of the small samples were determined separately from measurements of the parallel component of the magnetization.

The arc-melted buttons of the alloys, which had a thickness of 4-5 $\mathrm{mm}$ and diameter of $22-30 \mathrm{~mm}$, were turned and remelted five times before annealing. The homogeneity perpendicular to a button melted in this way has been investigated in Ref. 2 and was there found to vary by about $\pm 1 \%$. The concentrations determined by magnetization measurements in the present work of samples usually cut on different positions of the button and in different grains have shown inhomogeneities up to about $\pm 10 \%$ in the buttons as a whole.

\section{THEORETICAL PROCEDURE}

$\mathrm{Tb}$, Dy, and $\mathrm{Er}$ are expected to form tripositive ions when dissolved in $\mathrm{Y}$, with ground-state multiplets having total angular-momentum quantum numbers $J$ of $6, \frac{15}{2}$, and $\frac{15}{2}$, respectively. The effects of mixing of the $J$ multiplets by the crystal field were calculated for $\mathrm{Tb}$, which has the smallest energy separations between the $J$ multiplets. These effects were found to be negligible. In the analysis of the experimental data we therefore assumed that the crystal field does not mix the $J$ multiplets in the lowest $L S$ term. In this asumption the most general operator describing the crystal field for a $4 f$ ion in a hexagonal-closepacked lattice is

$$
\mathfrak{H}_{\mathrm{CF}}=B_{20} O_{20}+B_{40} O_{40}+B_{60} O_{60}+B_{66} O_{66} .
$$

The $O_{l m}{ }^{\prime}$ s are the Stevens operators ${ }^{4}$ and the $B_{l m}{ }^{\prime} \mathrm{s}$ are the crystal-field parameters. In (1) it is assumed that the coordinate axes $x, y$, and $z$ lie along the crystallographic axes $a, b$, and $c$, respectively. The total Hamiltonian used for the dilute alloys is

$$
\begin{aligned}
& \mathcal{H}=\mathcal{H}_{\mathrm{CF}}+g_{J} \mu_{B} \overrightarrow{\mathrm{J}} \cdot \overrightarrow{\mathrm{H}}_{\text {eff }}, \\
& \overrightarrow{\mathrm{H}}_{\text {eff }}=\overrightarrow{\mathrm{H}}+\underline{\lambda} \cdot \overrightarrow{\mathrm{M}} .
\end{aligned}
$$

In the Zeeman operator the small exchange interactions are included in the molecular-field approximation. $g_{J}$ is the Lande factor, $\mu_{B}$ the Bohr magneton, $\vec{J}$ the total angular momentum operator, $\overrightarrow{\mathrm{H}}$ the internal field, $\lambda$ the molecular-field tensor, and $\vec{M}$ the magnetization per rare-earth atom. $\underline{\lambda}$ has axial symmetry with components $\lambda_{11}$ and $\lambda_{\perp}$ in the $c$ and basal-plane directions, respectively. The magnetizations were calculated by diagonalizing the total Hamiltonian (2). The zero-field susceptibilities were calculated according to the method described in Ref. 1, which only involved a diagonalization of the crystal-field Hamiltonian (1).

The crystal-field parameters as well as $\lambda_{\|}$, $\lambda_{\perp}$ and the actual concentrations were obtained by a simultaneous least-squares fit to the reciprocal initial susceptibilities in the $c$ and $b$ directions, as described earlier. ${ }^{1}$ The sign of $B_{66}$ does not influence the initial susceptibility and had to be determined from separate measurements of the $a$ and $b$ axis magnetizations at high fields (VSM). For $\mathrm{Tb}$ it was not possible to determine the magnitude of $B_{66}$ from the susceptibility data alone. In this case the basal-plane hexagonal anisotropy measurements had to be included in the fit.

The perpendicular component $M_{\perp}$ of the basalplane magnetization for a paramagnetic crystal can be written

$$
\begin{aligned}
M_{\perp}(H, T, \varphi)= & M_{\perp, 6}(H, T) \sin 6 \varphi+M_{\perp, 12}(H, T) \\
& \times \sin 12 \varphi+\cdots
\end{aligned}
$$

$\varphi$ is the angle between the $a$ axis and the magnetic field which lies in the basal plane. $M_{\perp, 6}(H, T)$, which is the largest amplitude in (3) in the present experiment, was calculated and compared with experimental values. In order to obtain sufficient numerical accuracy it was convenient to use a real matrix representation of $\mathcal{H C}[\mathrm{Eq}$. (2)]. This was accomplished by choosing a coordinate system with the $z$ axis along $H_{\text {eff }}$ and the $y$ axis along the $c$ axis. In this frame of coordinates the crystalfield Hamiltonian $\mathcal{H}_{\mathrm{CF}}$ has a complicated form in terms of Stevens operators. ${ }^{5}$ A small angular deviation between the effective field and the internal magnetic field implies a correction to the value $M_{\perp}^{0}$, which is calculated by using the magnetic-moment operator $-g_{J} \mu_{B} J_{x}$. To first order in the exchange field

$$
\overrightarrow{\mathrm{H}}_{\mathrm{ex}}=\underline{\lambda} \cdot \overrightarrow{\mathrm{M}} \text {. }
$$



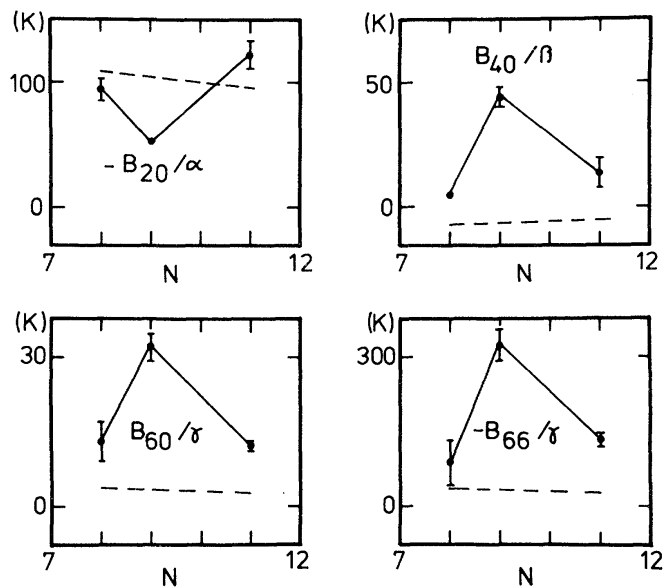

FIG. 2. Crystal-field parameters divided by the Stevens factors and plotted as a function of the number of $4 f$ electrons in the rare earths. The dashed curve is calculated in the point-charge model (Ref. 6) using the lattice parameters for pure Y. Tb: $N=8$; Dy: $N=9$; Er: $N=11$.

The corrected value is

$$
M_{\perp}^{\text {corr }}=M_{\perp}^{0}\left(1+\frac{H_{\mathrm{ex}}}{H}\right) \text {. }
$$

\section{EXPERIMENTAL RESULTS}

In this section we present a comparison between experimental results and our theoretical curves,
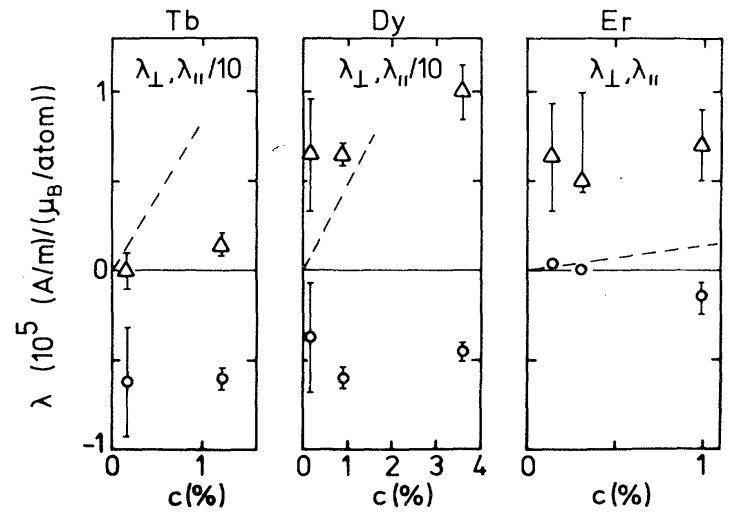

FIG. 3. Molecular-field parameters vs concentration. $\lambda_{1}: \Delta ; \lambda_{11}, \lambda_{11} / 10: 0$. The dashed lines are the isotropic part of the molecular-field tensor obtained from the paramagnetic Curie temperatures of the pure rare-earth metals (Ref. 7) multiplied by the concentration (see text).

which are calculated using the crystal-field and the molecular-field parameters summarized in Fig. 2 and in Fig. 3, respectively. In all experimental curves the $\mathrm{Y}$ background is subtracted. Magnetizations and susceptibilities are given per rare-earth atom.

$\mathrm{Tb}$

Of the three binary systems studied $\mathrm{Y}_{1-x} \mathrm{~Tb}_{x}$ has least structure in the inverse initial susceptibility

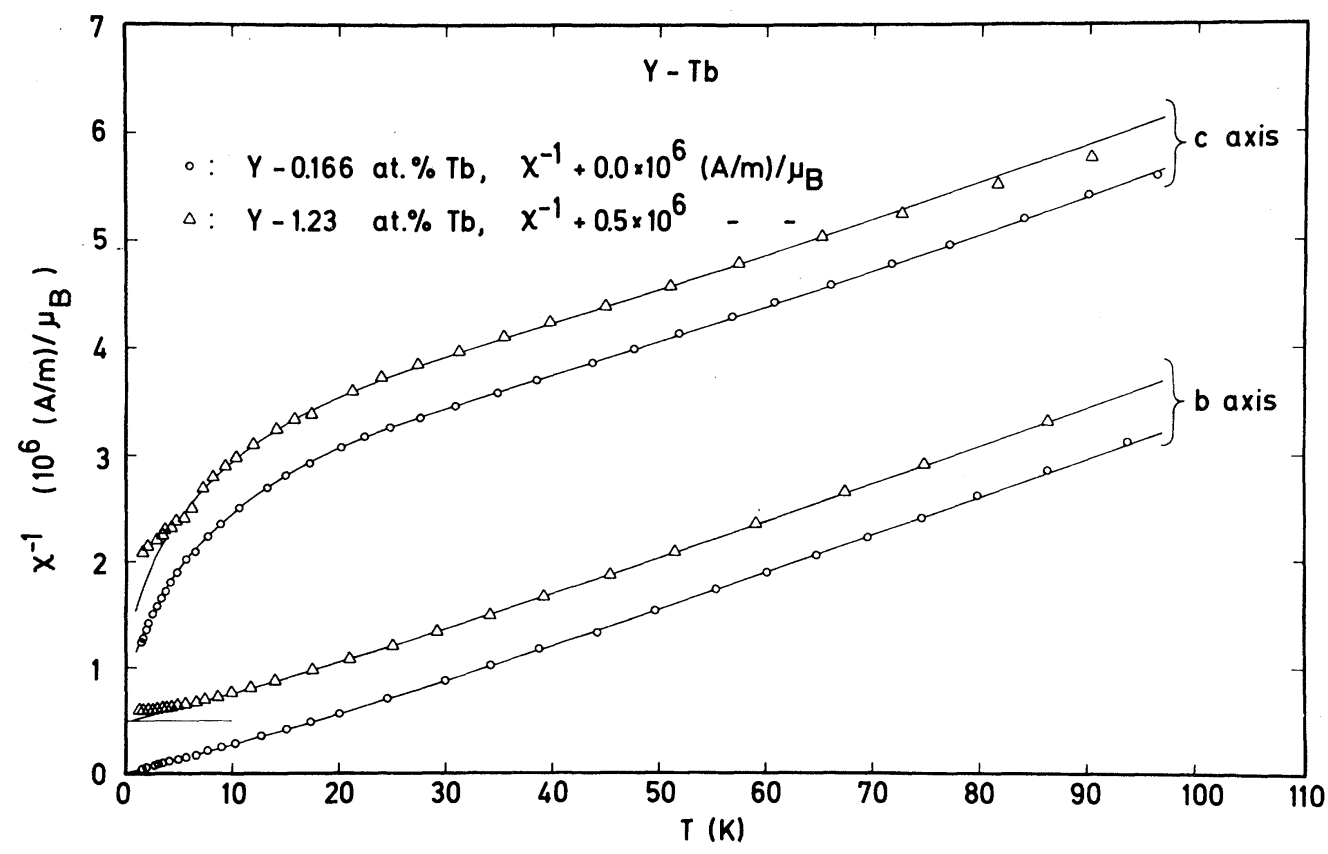

FIG. 4. Reciprocal susceptibilities for $\mathrm{Tb}$ and $\mathrm{Y}$. (FM at $\left.1.35 \times 10^{5} \mathrm{~A} / \mathrm{m}\right)$. The full curves are calculated. 
TABLE I. Crystal-field parameters in Kelvin.

\begin{tabular}{ccccrr}
\hline \hline $\mathrm{Y}_{1-x_{x} \mathrm{R}_{x}{ }^{\mathrm{a}}}$ & $0.140 \% \mathrm{Er}^{\mathrm{b}}$ & $0.309 \% \mathrm{Er}$ & $0.141 \% \mathrm{Dy}$ & $0.898 \% \mathrm{Dy}$ & $0.166 \% \mathrm{~Tb}$ \\
\hline$B_{20}$ & $-0.309 \pm 10 \%$ & $-0.298 \pm 10 \%$ & $0.321 \pm 10 \%$ & $0.336 \pm 6 \%$ & $0.945 \pm \mathbf{1 0} \%$ \\
$B_{40}$ & $0.814 \times 10^{-3} \pm 10 \%$ & $0.6 \times 10^{-3} \pm 40 \%$ & $-0.293 \times 10^{-2} \pm 10 \%$ & $-0.260 \times 10^{-2} \pm 10 \%$ & $0.589 \times 10^{-3} \pm 20 \%$ \\
$B_{60}$ & $0.243 \times 10^{-4} \pm 10 \%$ & $0.231 \times 10^{-4} \pm \mathbf{1 0} \%$ & $0.418 \times 10^{-4} \pm 10 \%$ & $0.335 \times 10^{-4} \pm 10 \%$ & $-0.145 \times 10^{-4} \pm 25 \%$ \\
$B_{66}$ & $-0.279 \times 10^{-3} \pm 10 \%$ & $-0.272 \times 10^{-3} \pm 10 \%$ & $-0.333 \times 10^{-3} \pm \mathbf{1 0} \%$ & $-0.334 \times 10^{-3} \pm 10 \%$ & $0.100 \times 10^{-3} \pm 25 \%$ \\
\hline
\end{tabular}

${ }^{\mathrm{a}} R=$ rare earth.

as a function of temperature (Fig. 4). As mentioned earlier, we have to include basal-plane anisotropy measurements (Fig. 5) in the fit to obtain all crystal-field parameters. The $1.23 \%-\mathrm{Tb}$ alloy is clearly seen to exhibit incipient ordering at $4.2 \mathrm{~K}$ (Fig. 4), and it could not be used for a separate determination of the crystal-field parameters. Using the crystal-field parameters obtained for $0.166 \% \mathrm{~Tb}$ (Table I) the molecular-field parameters and the actual concentration for $1.23 \%$ $\mathrm{Tb}$ were found by a fitting procedure. The variation of $M_{\perp}$ as a function of temperature and field (Fig. 5) could not be completely fitted due to ordering effects. In Fig. 5 theoretical curves are calculated for two different values of $B_{66}$ to illustrate the effects of varying this parameter. $M_{\|}$ measured in the $a$ and $b$ directions (Fig. 6) at

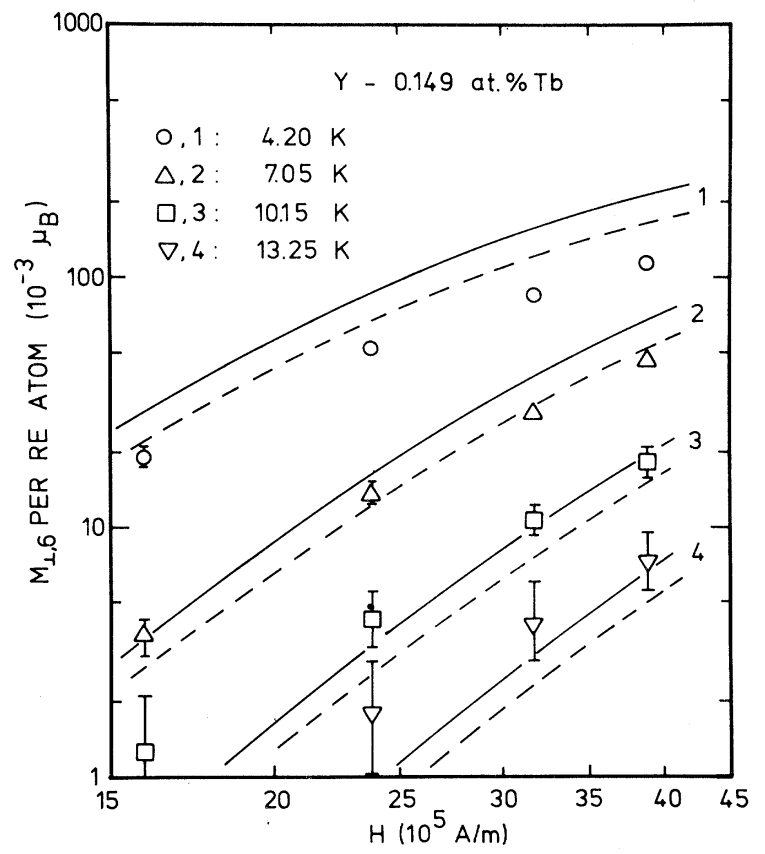

FIG. 5. Perpendicular basal-plane sixth-order component vs temperature and field for $0.149 \% \mathrm{~Tb}$ in $\mathrm{Y}$. The full and dashed curves are claculated using $\boldsymbol{B}_{66}=0.110$ $\times 10^{-3} \mathrm{~K}$ and $B_{66}=0.84 \times 10^{-4} \mathrm{~K}$, respectively.
$H=44.1 \times 10^{5} \mathrm{~A} / \mathrm{m}$ clearly shows the $b$ axis to be the axis of easy magnetization and also illustrates the importance of measuring $M_{\perp}$ for an accurate determination of the basal-plane anisotropy. The isothermal magnetization curves for the $0.166 \%$ $\mathrm{Tb}$ alloy are very well accounted for at higher temperatures but deviate from the theoretical curves at low temperatures (Fig. 7), demonstrating the effects of ordering at low temperatures even for this dilute alloy.

Dy

The characteristic features of the reciprocal zero-field susceptibilities for $\mathrm{Y}_{1-x} \mathrm{Dy}_{x}$ (Fig. 8) allow an accurate determination of the crystalfield parameters for these alloys. The crystalfield parameters for $0.14 \%$ Dy and $0.898 \%$ Dy agree to within the accuracy of their determination (Table I). The 3.60\%-Dy sample orders, and the data for this crystal are not adequate for a determination of the crystal-field parameters.

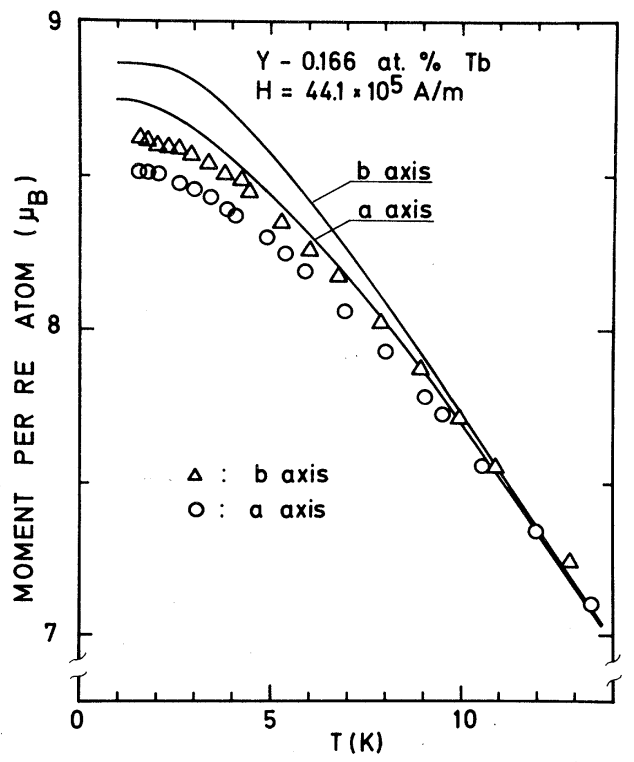

FIG. 6. Isofield curves in the $a$ and $b$ directions at $44.1 \times 1.0^{5} \mathrm{~A} / \mathrm{m}$ for $0.166 \% \mathrm{~Tb}$ in $\mathrm{Y}$. The full curves are calculated. 


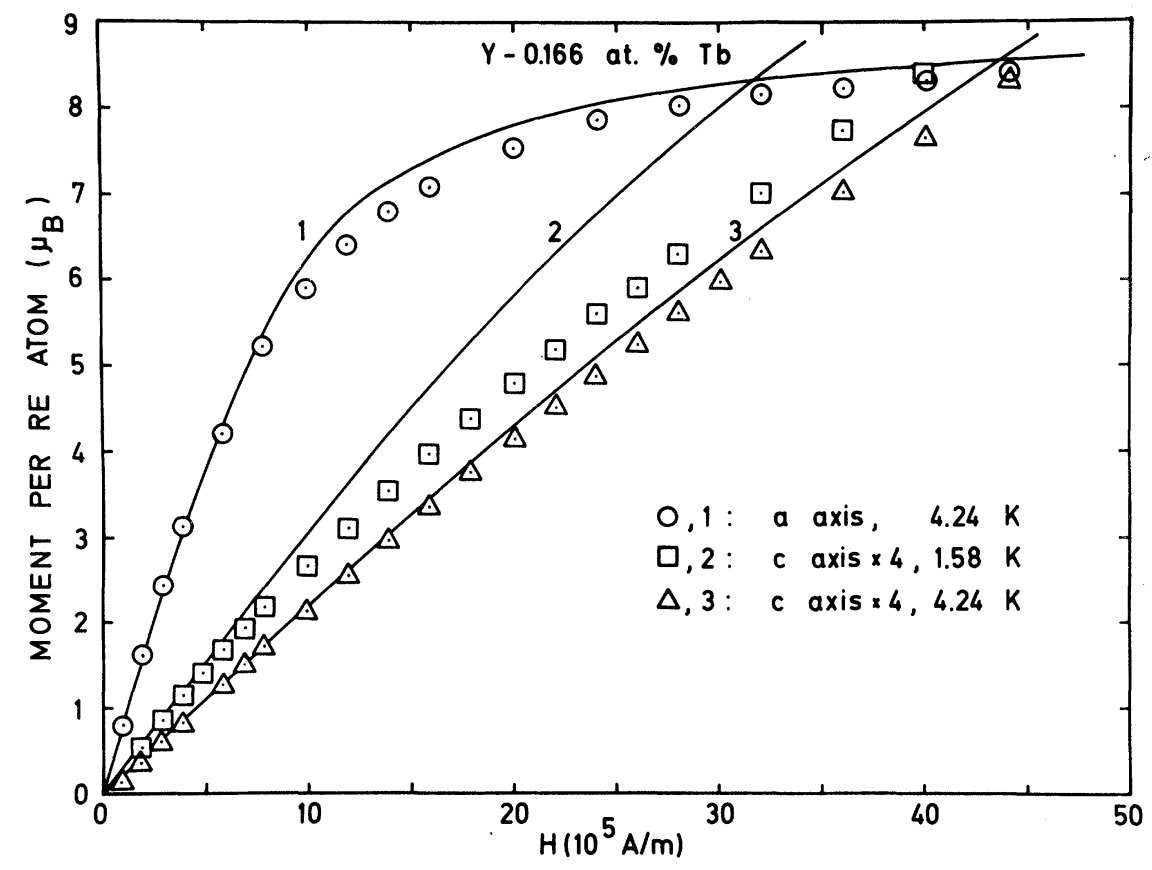

FIG. 7. Isotherms for $0.166 \% \mathrm{~Tb}$ in $\mathrm{Y}$. The full curves are calculated.
As the $\mathrm{Y}$ background in the $0.898 \%$-Dy alloy is less important than in the $0.141 \%$-Dy alloy, the crystalfield parameters obtained for the former are expected to be the most reliable. These parameters together with the molecular-field constants and the actual concentration found for each alloy separately are therefore used to calculate all curves in Fig. 8. Figure 9 shows isotherms for $3.60 \%$ Dy. The ordering at $8 \mathrm{~K}$ is clearly seen from the inset, which shows the reciprocal $b$-axis susceptibilities for different fields. Neutron-diffraction measurements ${ }^{8}$ on the same crystal have shown that it orders in a helical

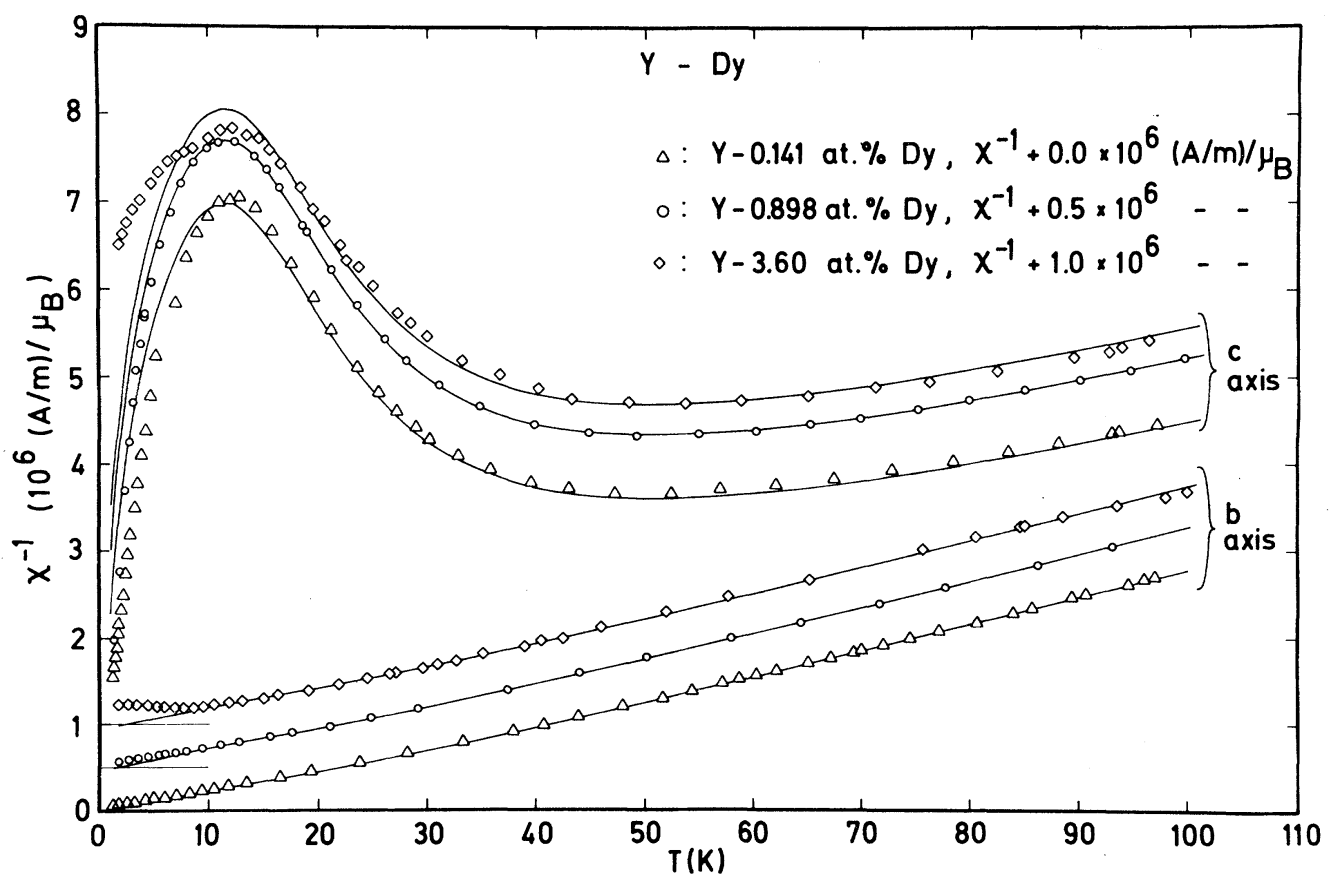

FIG. 8. Reciprocal susceptibilities for Dy in $\mathrm{Y}\left(\mathrm{FM}\right.$ at $\left.1.35 \times 10^{5} \mathrm{~A} / \mathrm{m}\right)$. The full curves are calculated. 


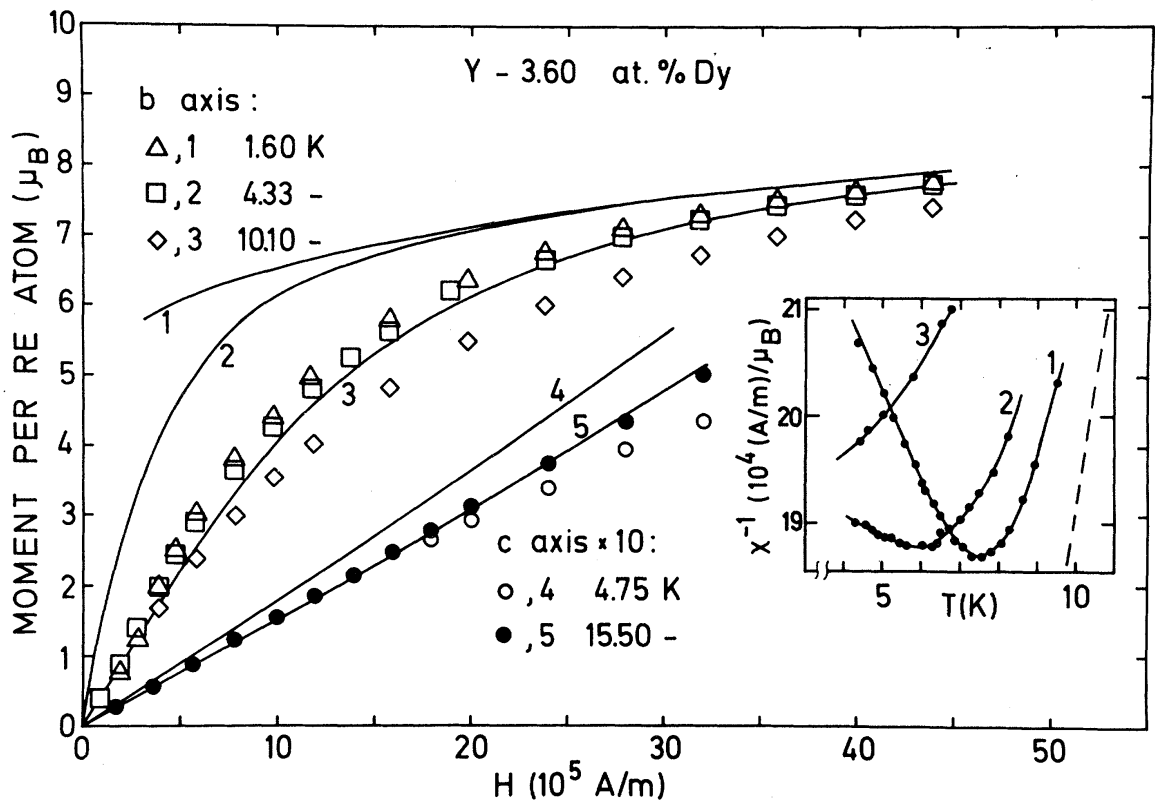

FIG. 9. Isotherms for $3.60 \%$ Dy in $\mathrm{Y}$. The full isothermal curves are calculated. The inset shows the reciprocal susceptibilities $H / M$ for $H\left(10^{5} \mathrm{~A} / \mathrm{m}\right)=1$ : $1.36,2: 2.71$, and $3: 5.80$. The dashed curve is calculated for $H=0$.

structure with the moments lying in the basal plane. This explains the large deviations below 8 $\mathrm{K}$ between the experimental data and the theoretical curves which are calculated using the $q=0$ molecular-field parameters (Figs. 8 and 9). We also found deviations in the $c$-axis magnetizations at low temperatures for the $0.898 \%$-Dy sample (Fig. 10) and even in the case of $0.141 \% \mathrm{Dy},{ }^{1}$ and we ascribe this to short-range-order effects. The importance of these effects is also emphasized by the measurements of the perpendicular component of the basal-plane magnetization, $M_{\perp}$, displayed in Fig. 11. The sixth-order Fourier component at $4.2 \mathrm{~K}$ is a smooth function of the field for $0.141 \%$ Dy. For $0.785 \%$ Dy it is still monotonic but it has a plateau around $12 \times 10^{5} \mathrm{~A} / \mathrm{m}$. This develops into a double change of sign for $3.72 \% \mathrm{Dy}$ and is accompanied by a large Fourier component of twelfth order (Fig. 12). In the antiferromagnetic phase, pure Dy shows similar changes of sign of the hexagonal anisotropy ${ }^{9}$ which is here attributed to the formation of the special fan structure treated theo-

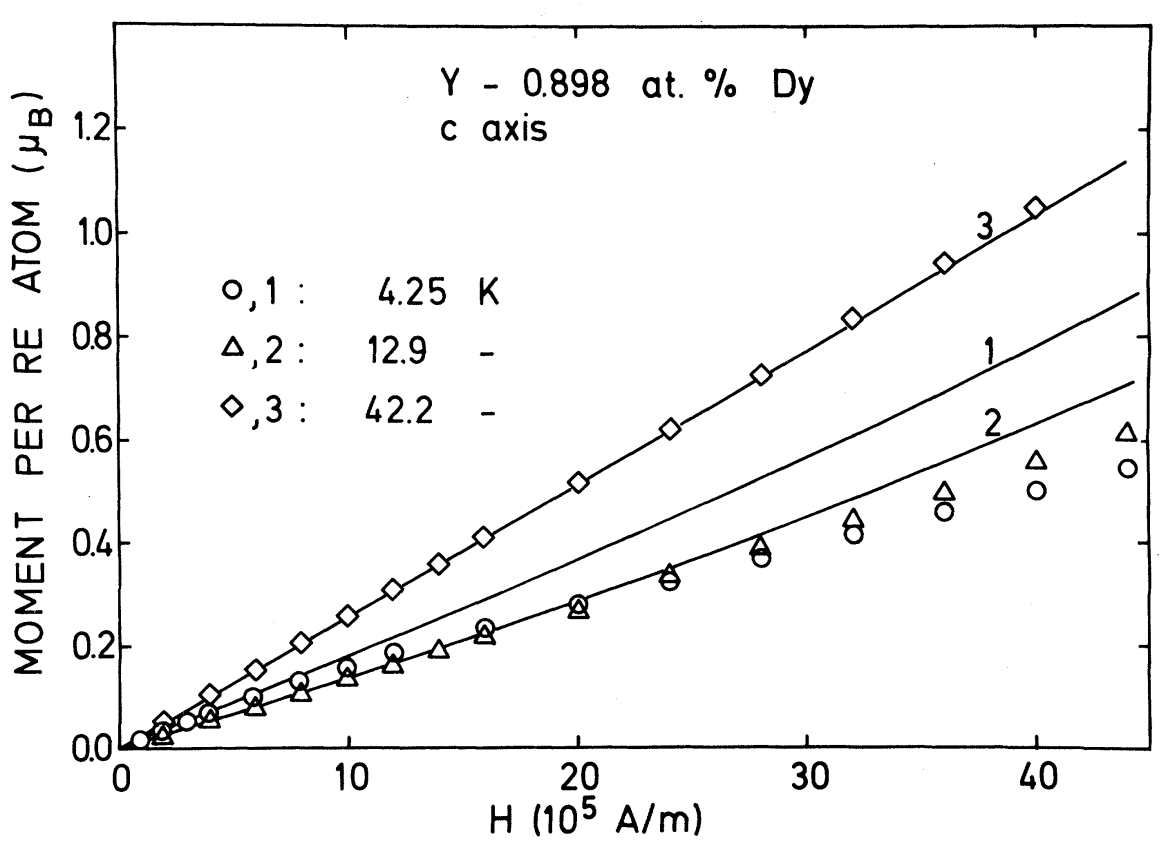

FIG. 10. Isotherms in the $c$ direction for $0.898 \%$ Dy in Y. The full curves are calculated. 


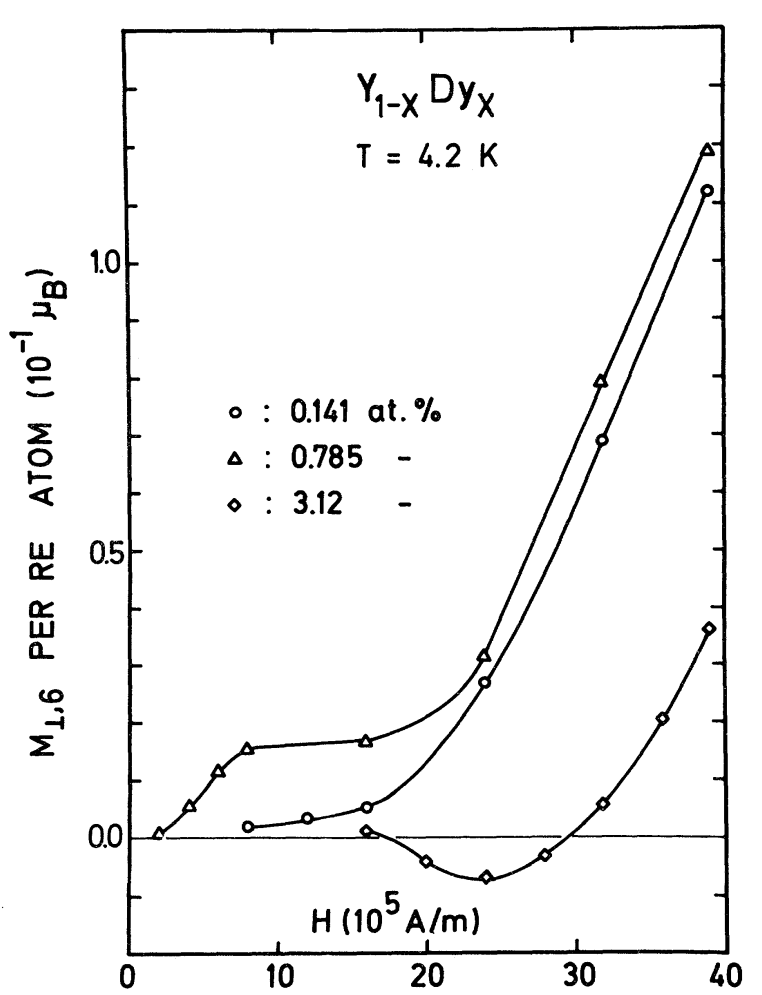

FIG. 11. Perpendicular basal-plane sixth-order component vs field for different concentrations of Dy in $\mathrm{Y}$ at $4.2 \mathrm{~K}$. The curves are drawn by hand.

retically by Nagamiya. ${ }^{10}$ In Fig. 13 the sixthorder harmonics for $0.141 \%$ Dy are shown for different temperatures together with calculated curves, which are seen to agree well with the experimental data.

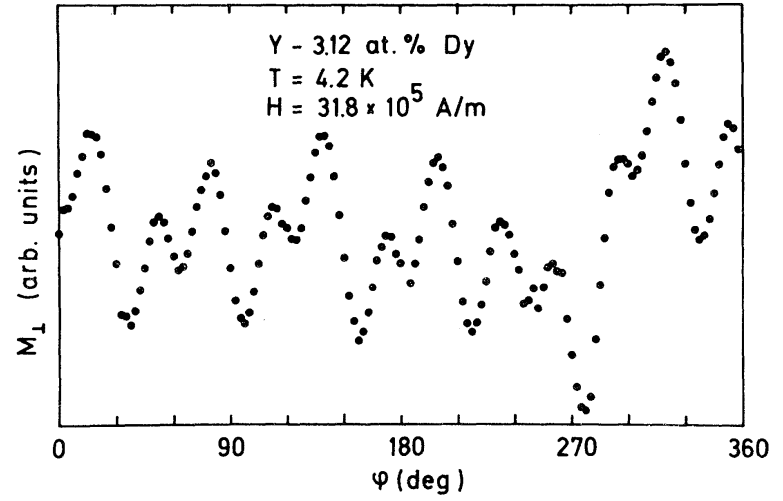

FIG. 12. VSA recording demonstrating a large $\sin (12 \varphi)$.

Er

In Fig. 14 the reciprocal susceptibilities for $\mathrm{Y}_{1-x} \mathrm{Er}_{x}$ are displayed. The curves allow a rather accurate determination of the crystal-field parameters. Except for $B_{40}$ the crystal-field parameters for $0.140 \% \mathrm{Er}$ and $0.309 \% \mathrm{Er}$ agree with the accuracy of their determination (Table I). $B_{40}$ is found to be less well determined than reported for the $0.140 \% \mathrm{Er}$, presumably due to systematic errors arising from impurities in this dilute alloy. $B_{40}$ plotted in Fig. 2 is the mean value for the two alloys, and the accuracy is chosen to cover both. The crystal-field parameters for $0.993 \% \mathrm{Er}$ are not included in Table I due to rather poor accuracy arising mainly from the small number of experimental points above $10 \mathrm{~K}$. The magnetization as a function of field and temperature is well described for all Er alloys as the results for $0.140 \% \mathrm{Er}$ in Ref. 1 illustrate. The small inconsistencies be-

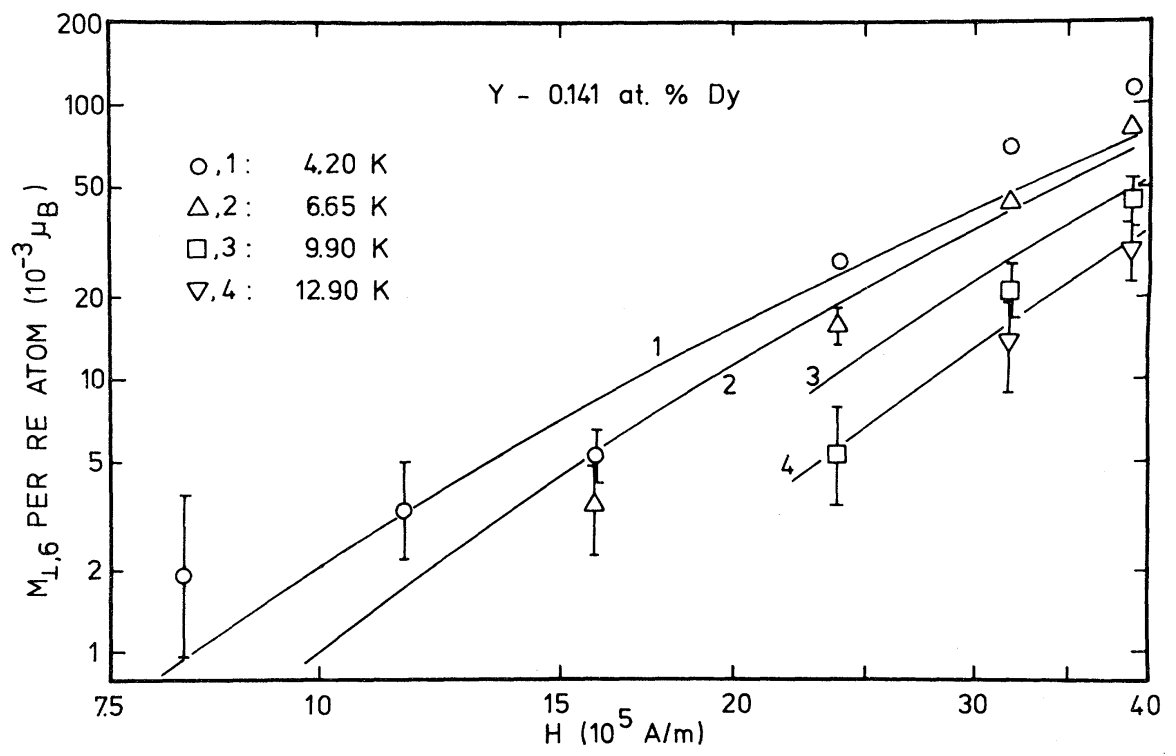

FIG. 13. Perpendicular basal-plane sixth-order component vs field and temperature for $0.141 \%$ Dy in Y. The full curves are calculated. 


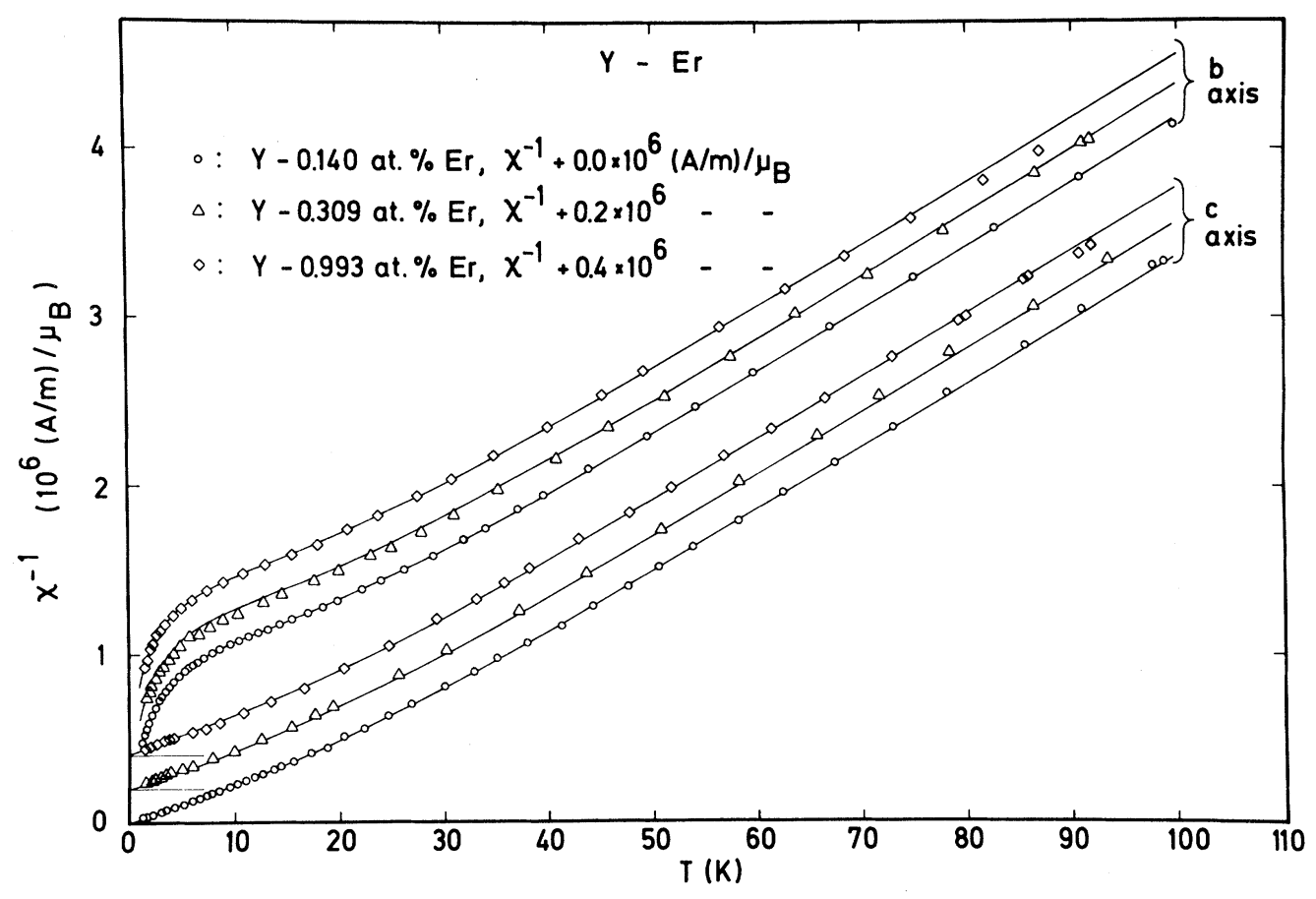

FIG. 14. Reciprocal susceptibilities for Er in Y. (FM at $\left.1.35 \times 10^{5} \mathrm{~A} / \mathrm{m}\right)$. The full curves are calculated.

tween the theoretical and experimental basal-plane anisotropy (Fig. 15) can be easily accounted for by the uncertainties in the crystal-field parameters. An inaccuracy of $10 \%$ on $B_{20}, B_{60}$, or $B_{66}$ results in an inaccuracy of about $25 \%$ in the theoretical curve at $4.2 \mathrm{~K}$. The influence of $B_{40}$ is ten times smaller.

\section{DISCUSSION}

The measurements of initial susceptibility, magnetization, and basal-plane anisotropy on alloys of different concentrations have supplied additional information on the use of such measurements for the determination of crystal-field and molecular-field parameters, and have confirmed the reliability of these parameters.

In the following we will discuss possible sources of error. In the Hamiltonian (2), we have neglected effects arising from the exchange interaction between the rare-earth impurity and the conduction electrons resulting in contributions to the magnetic moment due to localized electron polarization. This effect is important at low temperatures and is most clearly seen in the saturation magnetization of $0.140 \% \mathrm{Dy}^{1}$

Another effect which the Hamiltonian (2) does not describe is broadening of the crystal-field levels. The presence of broadening of full width at half-maximum values of $11 \mathrm{~K}$ have been observed in an inelastic-neutron-diffraction experiment on $\mathrm{Y}-2 \%-\mathrm{Er} .^{11}$ However, calculations have shown that broadenings of this magnitude only have significant effect on the initial susceptibility below about $5 \mathrm{~K} .{ }^{12}$

The third possible source of error is the simple model used for describing the exchange. This is most severe for the less dilute alloys where ordering or incipient ordering occurs, and in the worst cases $(1.23 \% \mathrm{~Tb}$ and $3.60 \%$ Dy) reasonably accurate crystal-field parameters could not be obtained from the fit to the inverse initial susceptibility curves. The basal-plane anisotropy is especially sensitive to ordering, and ordering effects were observed in even the most dilute alloys.

All these sources of error are only significant at low temperatures, and inconsistencies between theory and experiment are generally observed at low temperatures in susceptibility, magnetization, and basal-plane anisotropy curves. At higher temperatures we find excellent agreement between theory and experiment in all cases. This fact, together with the separate check which the basalplane anisotropy measurements give for $\operatorname{Er}$ and Dy, provides confidence in the parameters obtained. The Hamiltonian (2) also satisfactorily explains the variation of the susceptibility curves with concentration in terms of concentration-independent crystal-field parameters and concentration-dependent molecular-field parameters. The adequacy at higher temperatures of the molecular- 


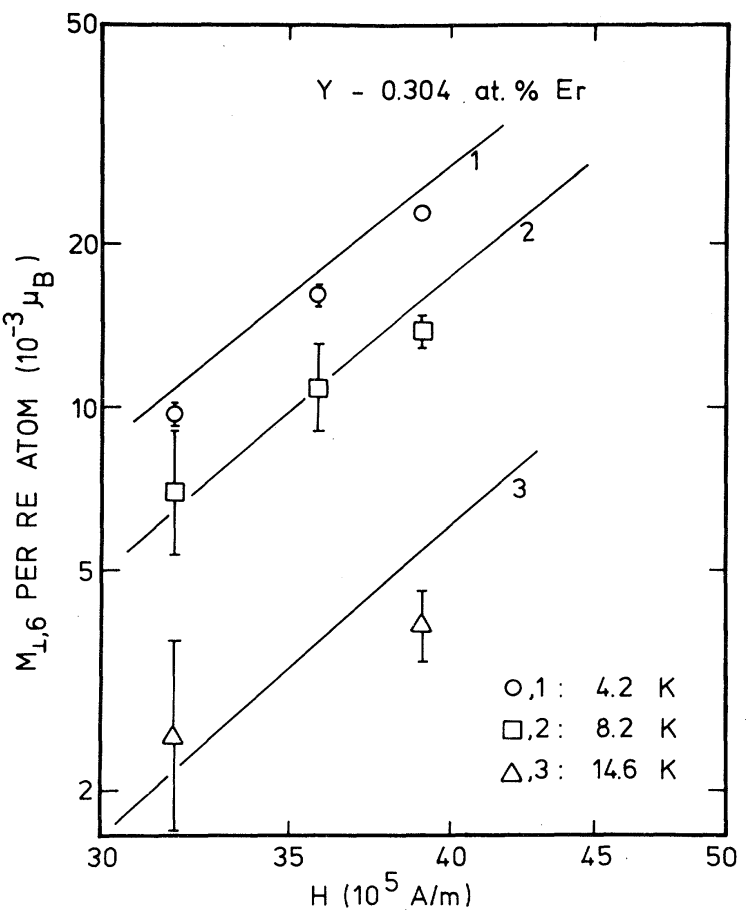

FIG. 15. Perpendicular basal-plane sixth-order component vs field and temperature for $0.304 \% \mathrm{Er}$ in $\mathrm{Y}$. The full curves are calculated.

field model, which only shifts the inverse initial susceptibility curves according to the relation

$$
1 / \chi=(1 / \chi)_{C F}-\lambda,
$$

is excellently demonstrated by the parallel curves in Figs. 4, 8, and 14.

In Fig. 2 the variation across the three rareearth metals of the crystal-field parameters divided by the Stevens factor $\mathrm{s}^{1,4}$ are illustrated. This picture supports the conclusion obtained in Ref. 1 about the inadequacy of the point-charge model and the importance of charges on the rare-earth ion itself in determining the crystal fields.

The crystal-field level schemes for $\mathrm{Tb}, \mathrm{Dy}$, and Er are given in Fig. 16, Ref. 1 (Fig. 12), and Fig. 17, respectively. The level scheme for Er given here is somewhat different for the one given in Ref. 1 (Fig. 11) due to the change of $B_{40}$ mentioned earlier. A high degree of accidental degeneracy is observed for $\mathrm{Tb}$. These level schemes are important for suggesting other kinds of experiment like ESR, neutron spectroscopy, and magnetization measurements in high fields to further elucidate the crystal fields. The possible neutron energy-loss transitions from the ground state doublet to the first, second, and third excited-state doublets in Er have been observed with correct energies and intensities within experi-

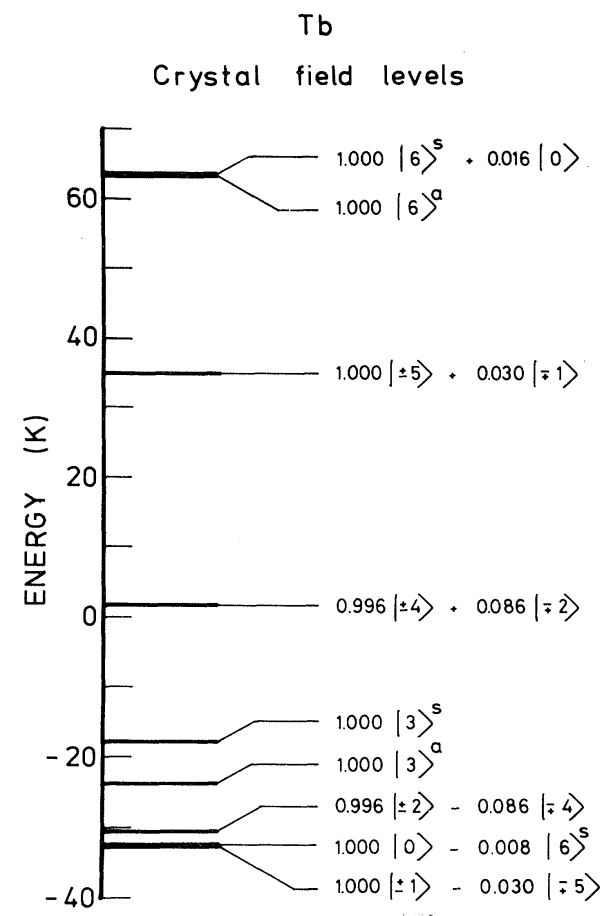

FIG. 16. Energies and eigenfunctions for $\mathrm{Tb}$ in $\mathrm{Y}$ calculated for $B_{20}=0.945, B_{40}=0.589 \times 10^{-3}, B_{60}=-0.145$ $\times 10^{-4}$, and $B_{66}=0.100 \times 10^{-3}$ (in Kelvin). $|m\rangle^{\mathrm{a}} \equiv(|m\rangle-$ $|-m\rangle) 2^{-1 / 2}$ and $|m\rangle^{s} \equiv(|m\rangle+|-m\rangle) 2^{-1 / 2}$.

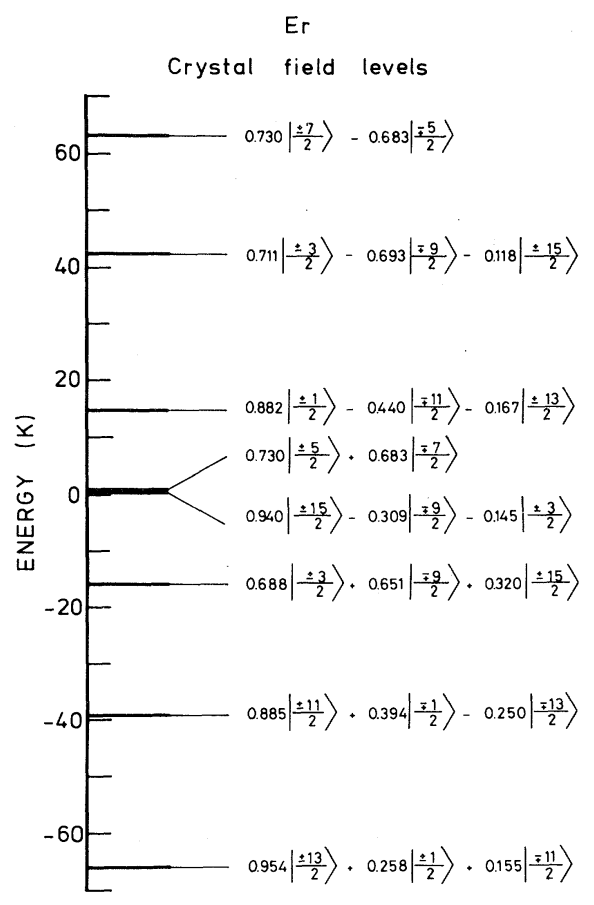

FIG. 17. Energies and eigenfunctions for $\mathrm{Er}$ in $\mathrm{Y}$ calculated for $B_{20}=-0.309, B_{40}=0.600 \times 10^{-3}, B_{60}=0.243$ $\times 10^{-4}$, and $B_{66}=-0.279 \times 10^{-3}$ (in Kelvin). 
mental accuracy. ${ }^{11}$ Characteristic features in the magnetizations-due to the crossing and mixing of crystal-field levels - have been observed in a high-field $\left(300 \times 10^{5} \mathrm{~A} / \mathrm{m}\right)$ experiment on $\mathrm{Y}-\mathrm{Tb}$, $\mathrm{Y}-\mathrm{Dy}$, and $\mathrm{Y}-\mathrm{Er}$ alloys. ${ }^{13}$ Also, these measurements have been satisfactorily accounted for by the parameters reported in the present work.

In Fig. 3 the molecular-field parameters defined in Eq. (2) are plotted versus concentration. A striking feature of this figure is the difference between $\lambda_{\perp}$ and $\lambda_{11}$, which even have opposite signs. The existence of highly anisotropic exchange interactions has also been observed by inelastic-neutron-diff raction experiments on pure $\operatorname{Er}^{14}$ and $\mathrm{Tb},{ }^{15}$ and is in contradiction with earlier models which assume isotropic exchange. For pure ion-ion exchange, simple theories predict the molecular-field parameters to be proportional to the concentration. In Fig. 3 the isotropic part of the molecular-field tensor obtained from the paramagnetic Curie temperatures of the pure rare-earth metals, ${ }^{7}$ and multiplied by the concentration, is also shown. $\lambda_{\perp}$ and $\lambda_{11}$ seem independent of the concentration at small concentrations, possibly reflecting contributions from conduction-electron exchange or from clustering. ${ }^{16}$ Due to the oscillatory and long-range character of the ion-ion indirect exchange in the rare-earth metals, any tendency for the rare-earth impurities to occupy sites in the lattice in a regular way will affect the molecular-field parameters.

\section{ACKNOWLEDGMENTS}

The authors are grateful to Dr. O. V. Nielsen, who kindly introduced them to the use of the vibrating sample anisotropy meter. They also wish to acknowledge fruitful discussions with Professor V. Frank and Professor A. R. Mackintosh. K. Pedersen assisted in the preparation of the singlecrystal samples.
${ }^{1} \mathrm{~J} . \mathrm{H} \phi \mathrm{g}$ and P. Touborg, Phys. Rev. B $\underline{9}, 2920$ (1974). ${ }^{2}$ K. A. McEwen and P. Touborg, J. Phys. F $\underline{3}, 1903$ (1973).

${ }^{3}$ O. V. Nielsen and V. I. Zaitzev, J. Phys. E $\underline{6}, 1022$ (1973).

${ }^{4}$ K. W. H. Stevens, Proc. Phys. Soc. Lond. A $\underline{65}, 209$ (1952).

${ }^{5}$ O. Danielsen and P. -A. Lindgård, Risö Report No. 259 (DK-4000 Roskilde, Denmark) (unpublished).

${ }^{6} \mathrm{~T}$. Kasaya, in Magnetism, edited by G. T. Rado and H. Suhl (Academic, New York, 1966), Vol. II B.

${ }^{7}$ P. Touborg, S. Balling, and K. M. Ravn (unpublished).

${ }^{8} \mathrm{O}$. Rathmann (private communication).

${ }^{9}$ P. H. Bly, W. D. Corner, and K. N. R. Tayler, J.
Appl. Phys. 40, 4787 (1969).

${ }^{10} \mathrm{~T}$. Nagamiya, in Solid State Physics, edited by F. Seitz, D. Turnbull, and H. Ehrenreich (Academic, New York, 1967), Vol. 20, p. 306.

${ }^{11}$ O. Rathmann, J. Als-Nielsen, P. Bak, J. Hфg, and P. Touborg, Phys. Rev. (to be published).

${ }^{12} \mathrm{P}$. Touborg and J. H $\phi \mathrm{g}$ (unpublished).

${ }^{13} \mathrm{P}$. Touborg, J. Hфg, G. J. Cock, and L. W. Roeland, Phys. Rev. B (to be published).

${ }^{14}$ R. M. Nicklow, N. Wakabayashi, M. K. Wilkinson, and R. E. Reed, Phys. Rev. Lett.

${ }^{15}$ J. Jensen, J. G. Houmann, H. Bjerrum Moller, and P. Touborg, Phys. Rev. B (to be published).

${ }^{16}$ The dipolar-dipolar interaction is negligible. 